\title{
Breast Angiomatosis
}

National Cancer Institute

\section{Source}

National Cancer Institute. Breast Angiomatosis. NCI Thesaurus. Code C40381.

A benign diffuse vascular proliferation in the breast. It is characterized by the formation of capillary-sized and cavernous vascular spaces. 